\section{(C) OPEN ACCESS}

\title{
Development and validation of a prediction rule for estimating gastric cancer risk in the Chinese high-risk population: a nationwide multicentre study
}

\author{
Quancai Cai, ${ }_{1}^{1}$ Chunping Zhu, ${ }^{1}$ Yuan Yuan, ${ }^{2}$ Qi Feng, ${ }^{3}$ Yichao Feng, ${ }^{4}$ Yingxia Hao, ${ }^{5}$ \\ Jichang Li, ${ }^{6}$ Kaiguang Zhang, ${ }^{7}$ Guoliang $\mathrm{Ye}^{8}{ }^{8}$ Liping $\mathrm{Ye}_{1}{ }^{9}$ Nonghua $\mathrm{Lv}_{1}{ }^{10}$ \\ Shengsheng Zhang, ${ }^{11}$ Chengxia Liu, ${ }^{12}$ Mingquan Li, ${ }^{13}$ Qi Liu, ${ }^{14}$ Rongzhou Li, ${ }^{15}$ \\ Jie Pan, ${ }^{16}$ Xiaocui Yang, ${ }^{17}$ Xuqing Zhu, ${ }^{18}$ Yumei Li, ${ }_{1}^{19}$ Bo Lao, ${ }^{20}$ Ansheng Ling, ${ }^{21}$ \\ Honghui Chen, ${ }^{22}$ Xiuling Li, ${ }^{23}$ Ping Xu, ${ }^{24}$ Jianfeng Zhou, ${ }^{25}$ Baozhen Liu, ${ }^{26}$ \\ Zhiqiang Du, ${ }^{27}$ Yiqi Du, ${ }^{\circ}$ Zhaoshen $\mathrm{Li}^{1}{ }^{1}$ Gastrointestinal Early Cancer Prevention \& \\ Treatment Alliance of China (GECA)
}

- Additional material is published online only. To view please visit the journal online (http://dx.doi.org/10.1136/ gutjnl-2018-317556).

For numbered affiliations see end of article.

Correspondence to Prof Yiqi Du and Prof Zhaoshen Li, Department of Gastroenterology, Changhai Hospital, Naval Medical University, Shanghai 200433, China; duyiqi@hotmail.com, zhsl@vip.163.com

QC, CZ and YY contributed equally.

Received 9 September 2018 Revised 8 March 2019 Accepted 12 March 2019 Published Online First 29 March 2019
Check for updates

(C) Author(s) (or their employer(s)) 2019. Re-use permitted under CC BY-NC. No commercial re-use. See rights and permissions. Published by BMJ.

To cite: Cai Q, Zhu C, Yuan Y et al. Gut

2019:68:1576-1587.

\section{ABSTRACT}

Objective To develop a gastric cancer (GC) risk prediction rule as an initial prescreening tool to identify individuals with a high risk prior to gastroscopy.

Design This was a nationwide multicentre crosssectional study. Individuals aged $40-80$ years who went to hospitals for a GC screening gastroscopy were recruited. Serum pepsinogen (PG) I, PG II, gastrin-17 (G-17) and anti-Helicobacter pylori lgG antibody concentrations were tested prior to endoscopy. Eligible participants ( $n=14929$ ) were randomly assigned into the derivation and validation cohorts, with a ratio of 2:1. Risk factors for GC were identified by univariate and multivariate analyses and an optimal prediction rule was then settled.

Results The novel GC risk prediction rule comprised seven variables (age, sex, PG I//I ratio, G-17 level, $H$. pylori infection, pickled food and fried food), with scores ranging from 0 to 25 . The observed prevalence rates of GC in the derivation cohort at low-risk $(\leq 11)$, medium-risk (12-16) or high-risk (17-25) group were $1.2 \%, 4.4 \%$ and $12.3 \%$, respectively $(p<0.001)$. When gastroscopy was used for individuals with medium risk and high risk, $70.8 \%$ of total GC cases and $70.3 \%$ of early GC cases were detected. While endoscopy requirements could be reduced by $66.7 \%$ according to the low-risk proportion. The prediction rule owns a good discrimination, with an area under curve of 0.76 , or calibration $(p<0.001)$.

Conclusions The developed and validated prediction rule showed good performance on identifying individuals at a higher risk in a Chinese high-risk population. Future studies are needed to validate its efficacy in a larger population.

\section{INTRODUCTION}

Gastric cancer (GC) is the second most common cancer in China. ${ }^{1}$ In 2015, an estimated 679100 persons were diagnosed with GC and 498000 persons eventually died of the disease in China, ${ }^{1}$ which accounts for approximately half of

\section{Significance of this study}

What is already known on this subject?

- Gastric cancer (GC) is one of the most common cancers worldwide, and nearly half of new cases and deaths in the world occur in China. Early detection and treatment can reduce the mortality of GC. Thus, secondary prevention (ie, early detection, diagnosis and treatment of GC) is important for GC.

- Although no nationwide screening programme is available in China, the current national screening guideline for GC in China recommends screening beginning at age 40 years for the $\mathrm{GC}$ risk population.

- With the Chinese GC risk population estimated to exceed 300 million individuals, it is not likely feasible to offer screening gastroscopy to all in this population, and thus, further risk stratification prior to gastroscopy is urgently needed. However, no such a tool is currently available in China.

What are the new findings?

- We developed and validated an applicable risk prediction rule to further stratify risk for GC in the Chinese GC risk population.

- The prediction rule identified $70.8 \%$ of individuals with $\mathrm{GC}$ and $70.3 \%$ of those with early GC among all participants and reduced gastroscopies by $66.7 \%$. Therefore, it has the potential to detect $\mathrm{GC}$ early and greatly reduce the costs associated with gastroscopy.

- The prediction rule had good performance and showed significantly better discrimination ability to identify a patient with GC than three other alternative prediction methods (ie, the $A B C$ method by Miki et al, ELISA-based ABC method in China and five biomarker-based method by Tu et al). 
Significance of this study

\section{How might it impact on clinical practice in the foreseeable future? \\ - The prediction rule could be used as an accurate, cost- effective and practical initial mass prescreening tool to identify a subgroup of individuals at higher risk prior to further diagnostic gastroscopy, which will improve the efficiency of GC detection in China.}

worldwide cases and deaths. ${ }^{2}$ The high mortality rate is mainly related to late detection, and thus, early detection and treatment are crucial to reduce deaths from GC. ${ }^{3}$ Therefore, there is an urgent need for a screening method to improve the detection of early-stage GC. ${ }^{4}$ However, at present, there is still no nationwide screening programme for GC in China, and early detection relies on opportunistic screening only. ${ }^{24-6}$

To change this situation, the current national screening guideline for GC in China recommends screening beginning at age 40 years for all in the high-risk population (ie, those residing in high-incidence areas for more than 3 years or who have Helicobacter pylori infection, a positive family history of GC, or risk factors for GC such as high-salt diet, smoking and heavy alcohol drinking). ${ }^{6}$ Gastroscopy with sampling of gastric biopsies for histological examination is currently the gold standard for screening and diagnosis of GC. However, screening the entire 'high-risk' population with gastroscopy is inefficient and impracticable as only $1 \%-3 \%$ of the population is expected to have GC. ${ }^{6}$ Moreover, with the estimated 'high-risk' population exceeding 300 million in China ${ }^{6}$ it is unlikely that screening gastroscopy can be offered to all this population due to the high costs. Therefore, a risk stratification method that can serve as an initial prescreening tool prior to gastroscopy is needed to further identify those real GC high-risk individuals among the previously defined 'high-risk' population.

Theoretically, a risk prediction rule for GC high-risk based on as many risk factors as possible, including age, sex, family history, high-salt diet, atrophic gastritis and $H$. pylori infection, rather than one subset of these risk factors, would provide better prescreening performance. However, to our knowledge, no such a prediction rule has been developed, most likely due to the fact that such a risk prediction rule is not cost-effective and feasible in clinical practice. Currently developed prescreening tools are mainly based on a subset of known risk factors for GC, specifically atrophic gastritis and $H$. pylori infection. ${ }^{7}$ Serum pepsinogen (PG) I, PG II and gastrin-17 (G-17) levels are used as surrogate biomarkers of atrophic gastritis in different topographic locations (PG I and PG II for the corpus; G-17 for the antrum), and anti-H. pylori IgG antibody is used for the detection of $H$. pylori infection. ${ }^{78}$

Miki ${ }^{9}$ developed the $\mathrm{ABC}$ method, which combines $H$. pylori serology with the measurement of serum PG levels, and demonstrated that it can predict future development of GC in Japan and thus identify individuals at high risk for future GC development. However, a few questions remain unanswered for the $\mathrm{ABC}$ method. The first question is whether the $\mathrm{ABC}$ method can be extended to predict current existence of GC and identity individuals at high risk for current GC existence. The second question is how this method would perform in prescreening for GC among the Chinese 'high-risk' population. Since previous studies have shown that the $\mathrm{ABC}$ method for defining atrophic PG status did not work well in the Chinese population. ${ }^{5910}$ The third question is what would be the optimal PG cut-off values for determining atrophic gastritis in China. Tu $e t a l^{5}$ evaluated a combination of five biomarkers, including serum PG I, PG II, PG I/II ratio, G-17 and anti-H. pylori IgG antibody in China, and their model showed better prescreening performance compared with the ABC method. However, the results may not applicable to the 'high-risk' population in China, as only individuals who had upper gastrointestinal symptoms or a positive family history of GC were included in the study. ${ }^{5}$

Therefore, the aim of this nationwide cross-sectional study was to develop a new prediction rule, for secondary prevention, to be used as an initial prescreening tool to identify a subgroup of individuals at higher risk of GC among the Chinese asymptomatic population for further diagnostic gastroscopy.

\section{METHODS}

\section{Study populations}

From 1 June 2015 to 31 March 2017, a total of 115 hospitals in China participated in this study. Individuals without gastrointestinal symptoms and aged between 40 and 80 years who came to the participating hospitals for a screening gastroscopy as part of a routine health check-up and met the criteria for Chinese individuals with a 'high risk' of GC were recruited into this study. The 'high-risk' for GC population among Chinese individuals was defined as persons who have lived in the areas of China with a high-incidence of GC for more than 3 years or who have a history of $H$. pylori infection, a positive family history of GC or risk factors for GC such as a highsalt diet, regular intake of pickled food, smoking and heavy alcohol drinking. ${ }^{6}$ A Chinese high-incidence area refers to a district where the incidence of GC is more than 30/100 000 . Participants were excluded from this study if they had one of the following symptoms or clinical findings: upper abdominal mass, unintentional weight loss of more than $4.5 \mathrm{~kg}$ within the previous 6 months, dysphagia, abdominal pain, melena, haematemesis and emesis. Other exclusion criteria were previous gastric resection; a history of $H$. pylori eradication; treatment with proton pump inhibitors or $\mathrm{H}_{2}$ blockers within the previous 2 weeks; impairment of renal function; pregnancy; a history of cancer of any type; a gastroscopy examination within 1 year before referral; and a medical condition that could increase the risk associated with gastroscopy.

\section{Study procedures}

All eligible subjects first completed a questionnaire, then provided fasting blood samples for serum determination of PG, G-17 and anti-H. pylori IgG antibody concentrations, and finally underwent gastroscopy.

\section{Questionnaire survey}

A prevalidated self-reported questionnaire was used in the present study. The questionnaire included baseline information (age, sex, body weight and height), living styles (smoking [smoke more than one cigarette every day for more than 1 year; if yes, the number of cigarette and lasting time were asked], alcohol consumption [drink alcohol of any types more than once every week lasting for more than 1 year; if yes, the kind of wine and consumption frequency were also asked], eating habits (high-salt diet [consume salt more than $10 \mathrm{~g} /$ day], pickled food, fried food, smoked food, barbecue food, overnight leftovers, red meat, white meat, green vegetables and fresh fruit [frequency was defined as more than three times per week]) and family history of GC among first-degree relatives (online supplementary file 1). 


\section{Laboratory methods}

A $5 \mathrm{~mL}$ fasting venous blood sample was collected from each eligible subject. After centrifugation, serum aliquots were stored at room temperature $\left(\leq 25^{\circ} \mathrm{C}\right)$ and immediately assayed within 3 hours. Serum concentrations of PG I, PG II, G-17 and anti-H. pylori IgG antibody were measured using commercial ELISA kits (PG I ELISA, PG II ELISA, G-17 ELISA and H. pylori IgG ELISA kits; Biohit, Helsinki, Finland) on a microplate reader (MB-580, Huisong Co, Shenzhen, China) by uniformly trained personnel in the qualified laboratories of all participating hospitals. The tests were performed in triplicate. Seropositivity for anti-H. pylori antibody was defined as anti- $H$. pylori IgG titre $\geq 34$ enzyme-immunoassay units according to the manufacturer's protocol.

\section{Cut-offs}

The cut-off values for PGs and G-17 were established according to the following steps: original concentrations in the non-GC population of derivation cohort were first logarithmically transformed and divided equally into 20 parts to obtain 20 cut-off values; then the prevalence of GC for each of the 20 cut-offs was calculated and the cut-off categories with similar prevalence rates were combined, two final categories for PGs and three categories for G-17; and finally, the antilog of the cut-off values of the combined categories were calculated and designated as the cut-off values of PGs and G-17, respectively.

\section{Gastroscopic examination}

In addition to white light gastroscopy, any further available examination in each hospital, including narrow band imaging, Fuji intelligent chromoendoscopy, i-Scan, blue laser imaging, magnifying endoscopy, confocal laser endomicroscopy, fluorescence endoscopy and chromoendoscopy, was required. Gastroscopy was performed at each hospital by expert endoscopists who had performed endoscopy examination or therapy for more than 1500 cases.

\section{Histological examination}

Gastric biopsy specimens were obtained from the gastric body, angulus, antrum and lesion location, if applicable, according to the Consensus on Screening and Endoscopic Diagnosis and Treatment of Early GC in China (2014 version). ${ }^{6}$ At least three biopsy specimens were collected from each participant according to the study protocol. Each histological specimen was independently reviewed by two pathologists. Gastric inflammation and atrophy were diagnosed according to the Updated Sydney System, ${ }^{11}$ and gastric tumours were diagnosed using the criteria from the Vienna classification for gastrointestinal epithelial neoplasia. ${ }^{12} 13$ Disagreement was solved by discussion; the final decision was made by the director of the Department of Pathology from the principal investigator's hospital if the disagreement remained. Participants with multiple lesions were categorised according to the most severe lesion. All participating endoscopists and pathologists were uniformly trained and performed the analyses according to the standard protocol.

The four procedures (ie, questionnaire, laboratory tests, gastroscopy and histology) were performed independently by different investigators who were blinded to the results of the each other examinations.

\section{Statistical analysis}

A central data library was established to manage the data from all participating hospitals in this study (on the website of www.ncrcgastro.org). Each hospital uploaded the data individually, and the supervisor in the leading site checked the integrity and accuracy of the data. Only once all requested data for one participant were successfully entered into the platform was the participant considered as a valid case to be included in the database for further analysis.

Two-thirds of the eligible participants were randomly assigned to the derivation cohort, while the remaining one-third was assigned to the validation cohort. Details on the development and validation of a prediction rule for estimating cancer risk were previously described. ${ }^{14}$ Gastroscopy with sampling of gastric biopsies was defined as the gold standard for definitive diagnosis. Participants found to have oesophageal cancer were excluded from further analyses. IBM SPSS Statistics for Windows (V.19.0) and R for Windows (V.3.5.1) were used for all statistical analyses. A two-sided $\mathrm{p}$ value of less than 0.05 indicated statistical significance.

In the derivation cohort, univariate and multivariate analyses were used to test associations of potential risk factors with GC. The potential risk factors with $\mathrm{p}<0.25$ in the univariate analyses were entered as independent variables into a binary logistic backward stepwise regression analysis to assess association with GC. To obtain an optimal prediction model, a new logistic regression model was developed by entering a different set of variables one at a time. The model's overall performance was assessed by $\mathrm{R}^{2}$ and Brier scores, and its discrimination was evaluated by the area under the receiver operating characteristic curve (AUC) and discrimination slope. Calibration was assessed by the Hosmer-Lemeshow $\chi^{2}$ statistic and calibration in the large. A regression coefficient-based scoring method was employed to develop the score-based prediction rule. ${ }^{14} 15$ In addition to overall performance, discrimination and calibration, the performance of the prediction rule was assessed according to its accuracy, including sensitivity, specificity, accuracy rate, positive predictive value (PPV), negative predictive value (NPV), positive likelihood ratio and negative likelihood ratio. The number needed to screen (NNS) was defined as the number of participants who would need to undergo gastroscopy for one patient with GC to be identified.

The prediction rule was internally validated by bootstrap resampling that used 1000 random samples drawn with replacement from the original derivation data set. ${ }^{16}{ }^{17}$ External validation was performed in the validation cohort to analyse the same performance metrics, including overall performance, discrimination, calibration and accuracy. The $u$ test was used to compare the difference in the AUCs between the derivation cohort and validation cohort.

The performance of the prediction rule, as assessed by AUC, was also compared against the $\mathrm{ABC}$ method $^{9}$ and a risk score by Tu et $a l^{5}$ in the validation cohort.

\section{RESULTS}

During the study period, 14929 eligible participants, including 6357 participants with normal gastric mucosa or non-atrophic gastritis, 405 patients with GC, 826 patients with low-grade intraepithelial neoplasia, 3651 patients with atrophic gastritis, 694 patients with gastric ulcer, 1872 patients with gastric polyps, 470 patients with duodenal ulcer, 78 patients with Barrett's oesophagus and 576 patients with reflux oesophagitis, were included in our analyses (online supplementary figure 1). Their mean (SD) age was 56.2 (9.6) years, and 49.7\% were men. Among the 405 patients with GC, 360 (88.9\%) had non-junctional cancer. There were 9838 participants in the derivation cohort, and $267(2.7 \%)$ of these participants had GC. The validation cohort contained 5091 participants, of whom 138 (2.7\%) had GC(figure 1). 


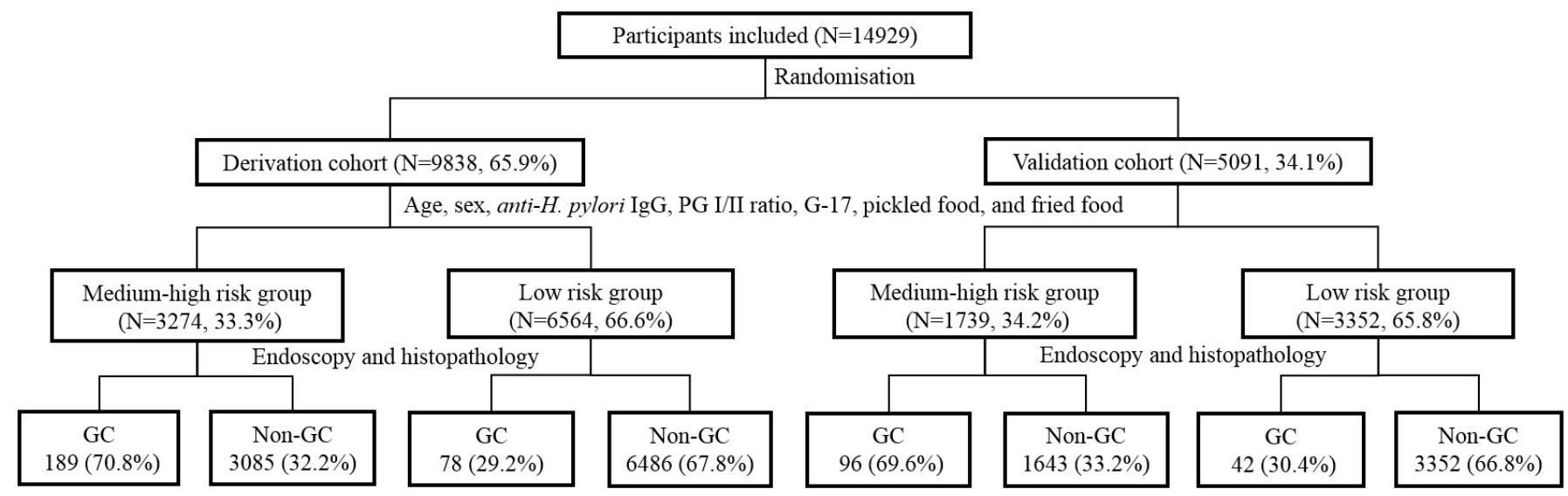

Figure 1 Flow chart showing the proposed algorithm for the clinical pathways for the respective individuals. The cut-off values for each factor are shown in tables 2 and 4. G-17, gastrin-17; GC, gastric cancer; PG, pepsinogen.

\section{Prediction rule development}

In the univariate analysis, 17 variables were found to be potentially associated with GC $(\mathrm{p}<0.25)$ and thus included in the multivariate analysis. Of the 17 variables, age, sex, PG I/II ratio, G-17 concentration, anti-H. pylori IgG status, regular consumption of pickled food and regular consumption of fried food were shown to be significantly (all p $<0.05$ ) and independently associated with the risk of GC (table 1 and online supplementary table 1). The regression model was used to develop the score-based prediction rule, which comprised seven variables (ie, age, sex, PG I/II ratio, G-17 concentration, anti-H. pylori IgG status, pickled food and fried food), with scores ranging from 0 to 25 (table 2). The prediction rule had good overall performance, with an $\mathrm{R}^{2}$ of 0.024 and a Brier score of 0.026 and showed good discrimination for GC, with an AUC of 0.76 (95\% CI 0.73 to 0.79) and good discrimination slope of 0.033 (95\% CI 0.033 to 0.035 ). Also, the model was well calibrated ( $\mathrm{p}=0.605$ by Hosmer-Lemeshow; $\mathrm{p}<0.001$ by calibration in the large; table 3 ).

As the score increased, the risk of GC increased. On the basis of similar magnitudes of risk and the result from receiver operating characteristic curve analysis of the prediction rule, scores 0 to 11,12 to 16 and 17 to 25 were combined to form three categories, corresponding to low, medium and high risk, respectively (table 4). Accordingly, in the derivation cohort, $66.7 \%$ of the participants were considered to be at low risk, whereas $27.6 \%$ and $5.7 \%$ were at medium and high risk, respectively. The prevalence rates of GC at low, medium or high risk were $1.2 \%, 4.4 \%$ and $12.3 \%$, respectively $(\mathrm{p}<0.001)$. When 12 was applied as the cut-off score for the prediction rule to identify individuals at medium or high risk, the sensitivity, specificity, PPV, NPV and accuracy were 70.8\%, 67.8\%, 5.8\%, 98.8\% and $67.9 \%$, respectively (table 3 ). Overall, individuals at medium or high risk accounted for $70.8 \%$ of all cases with GC. Early GC was detected in 30, 48 and 23 individuals at low, medium and high risk, respectively; $70.3 \%$ of cases with early GC were detected in individuals at medium and high risk, and $37.6 \%$ of GC cases detected in individuals at medium and high risk were in early stage. The NNSs in all individuals, those at low risk, and those at medium or high risk were 37,84 and 17 , respectively.

Since junctional and non-junctional GC have distinct risk factors, we further developed prediction models for junctional and non-junctional GC. We found that serum biomarkers (ie, PG I/PG II ratio and G-17) were included in the model for non-junctional GC but not in the model for junctional GC (online supplementary tables 2 and 3). In other words, the developed model performed well for non-junctional GC, but not for junctional GC.
In addition, intestinal-type and diffuse-type GC have distinct risk factors, so we also further developed prediction models for these two types. We found that the same seven factors were included in the model for intestinal-type GC, but only age was included in the model for diffuse-type GC (online supplementary tables 4 and5).

\section{Internal and external validation}

The performance metrics obtained from internal validation were the same as those from the derivation cohort (table 4). In the validation cohort, the risk of GC also increased as the score increased (table 4), and the respective risks of GC in individuals at low, medium and high risk were similar in magnitude to the risk estimates in the derivation cohort. The performance metrics in the validation cohort did not differ statistically from those in the derivation cohort ( $p>0.05$, table 3$)$. Similar to the findings in the derivation cohort, individuals at medium or high risk accounted for $69.6 \%$ of cases with GC, of which $31.3 \%$ were in early stage (the proportion of cases with early GC in these categories accounted for $61.2 \%$ of all cases with early GC). The respective NNS in all individuals, those at low risk, and those at medium or high risk were 37,80 and 18 , respectively, and these numbers were similar to those in the derivation cohort.

\section{Performance comparison with alternative prediction methods}

Among the four prediction methods that were assessed in the validation cohort, the prediction rule developed from the present study had the best discrimination (AUC: 0.757, $95 \%$ CI 0.729 to 0.786 , table 5). In contrast, three other prediction methods, including the $\mathrm{ABC}$ method by Miki, ${ }^{9}$ the ELISA-based $\mathrm{ABC}$ method in China ${ }^{5}{ }^{6}$ and the five biomarker-based method by Tu et al, ${ }^{5}$ displayed significantly lower AUCs compared with that of the prediction rule developed in the present study $($ all $\mathrm{p}<0.001)$.

\section{DISCUSSION}

In the present study, a new score-based prediction rule, which comprised the variables age, sex, PG I/II ratio, G-17 concentration, anti-H. pylori IgG status, consumption of pickled food and fried food, was developed and validated to provide accurate risk stratification for further GC screening in the Chinese population for secondary prevention of GC. The prediction rule had good performance both in the derivation cohort and validation cohort and showed significantly better discriminative ability to identify a patient at a high GC risk than other alternative prediction methods. Therefore, for the Chinese high-risk population, the 
Stomach

Table 1 Univariate and multivariable analyses in the derivation cohort

\begin{tabular}{|c|c|c|c|c|c|c|}
\hline Variable & $\begin{array}{l}\text { Total cohort } \\
(n=9838) \text { No. }\end{array}$ & $\begin{array}{l}\text { Gastric cancer } \\
(\mathrm{n}=267) \text { No. }(\%)\end{array}$ & $\begin{array}{l}\text { Non-gastric cancer } \\
(\mathrm{n}=9571) \text { No. }(\%)\end{array}$ & P value* & $\begin{array}{l}\text { Adjusted OR } \\
(95 \% \mathrm{Cl}) \dagger\end{array}$ & $P$ value \\
\hline Age, years & & & & $<0.001$ & & \\
\hline $40-49$ & 2741 & $22(8.2)$ & $2719(28.4)$ & & Reference & \\
\hline $50-59$ & 3457 & $79(29.6)$ & $3378(35.3)$ & & 2.77 (1.72 to 4.47$)$ & $<0.001$ \\
\hline $60-69$ & 2640 & $94(35.2)$ & $2546(26.6)$ & & 4.31 (2.69 to 6.89$)$ & $<0.001$ \\
\hline$>69$ & 1000 & $72(27.0)$ & $928(9.7)$ & & $8.67(5.32$ to 14.13$)$ & $<0.001$ \\
\hline Mean (SD) & $56.2(9.6)$ & $62.9(9.5)$ & $56.1(9.5)$ & $<0.001$ & & \\
\hline \multicolumn{7}{|l|}{ Body mass index $\ddagger$} \\
\hline Mean (SD) & $22.89(2.85)$ & $22.34(2.91)$ & $22.91(2.85)$ & 0.075 & & \\
\hline $\mathrm{PG} \mathrm{I}, \mathrm{ng} / \mathrm{mL}$ & & & & 0.048 & & \\
\hline$\leq 41.92$ & 597 & $25(9.4)$ & $572(6.0)$ & & & \\
\hline $41.93-277.00$ & 8651 & $223(83.5)$ & $8428(88.1)$ & & & \\
\hline$>277.00$ & 590 & $19(7.1)$ & $571(6.0)$ & & & \\
\hline Median (range) & $109.30(927.99)$ & $129.30(532.39)$ & 108.89 (927.99) & 0.001 & & \\
\hline PG II, ng/mL & & & & 0.008 & & \\
\hline$\leq 2.78$ & 466 & $11(4.1)$ & $455(4.8)$ & & & \\
\hline $2.79-37.23$ & 8806 & $229(85.8)$ & 8577 (89.6) & & & \\
\hline$>37.23$ & 566 & $27(10.1)$ & $539(5.6)$ & & & \\
\hline Median (range) & $9.20(856.66)$ & $13.90(215.45)$ & $9.10(856.66)$ & $<0.001$ & & \\
\hline PG I/II ratio & & & & $<0.001$ & & \\
\hline$\geq 3.89$ & 9235 & $227(85.0)$ & $9008(94.1)$ & & Reference & \\
\hline$<3.89$ & 603 & $40(15.0)$ & $563(5.9)$ & & $2.02(1.41$ to 2.90$)$ & $<0.001$ \\
\hline Median (range) & $11.84(869.13)$ & $8.38(103.33)$ & $11.95(869.13)$ & $<0.001$ & & \\
\hline \multicolumn{7}{|l|}{$\mathrm{G}-17, \mathrm{pmol} / \mathrm{L}$} \\
\hline$\leq 1.49$ & 2812 & $34(12.7)$ & $2778(29.0)$ & & Reference & \\
\hline $1.50-5.70$ & 3320 & $83(31.1)$ & 3237 (33.8) & & $2.00(1.33$ to 3.00$)$ & 0.001 \\
\hline$>5.70$ & 3706 & $150(56.2)$ & $3556(37.2)$ & & 2.84 (1.93 to 4.17$)$ & $<0.001$ \\
\hline Median (range) & $3.53(367.06)$ & 6.89 (99.99) & $3.48(367.06)$ & $<0.001$ & & \\
\hline Sex & & & & $<0.001$ & & \\
\hline Female & 4955 & $78(29.2)$ & $4877(51.0)$ & & Reference & \\
\hline Male & 4883 & $189(70.8)$ & $4694(49.0)$ & & 2.52 (1.92 to 3.30$)$ & $<0.001$ \\
\hline Anti-H. pylori IgG, EIU & & & & 0.028 & & \\
\hline Negative $(<34)$ & 5549 & $133(49.8)$ & $5416(56.6)$ & & Reference & \\
\hline Positive ( $\geq 34$ ) & 4289 & $134(50.2)$ & $4155(43.4)$ & & $1.26(1.12$ to 1.62$)$ & 0.046 \\
\hline Family history & & & & 0.856 & & \\
\hline No & 8657 & $234(87.6)$ & $8423(88.0)$ & & & \\
\hline Yes & 1181 & $33(12.4)$ & $1148(12.0)$ & & & \\
\hline Smoking & & & & $<0.001$ & & \\
\hline No & 7671 & $178(66.7)$ & 7493 (78.3) & & & \\
\hline Yes & 2167 & $89(33.3)$ & $2078(21.7)$ & & & \\
\hline Alcohol drinking & & & & 0.063 & & \\
\hline No & 8220 & $212(79.4)$ & 8008 (83.7) & & & \\
\hline Yes & 1618 & $55(20.6)$ & $1563(16.3)$ & & & \\
\hline High-salt diet & & & & 0.005 & & \\
\hline No & 5859 & $137(51.3)$ & $5722(59.8)$ & & & \\
\hline Yes & 3979 & $130(48.7)$ & $3849(40.2)$ & & & \\
\hline Pickled food & & & & $<0.001$ & & \\
\hline Occasional & 8070 & $196(73.4)$ & $7874(82.3)$ & & Reference & \\
\hline Regular & 1768 & $71(26.6)$ & 1697 (17.7) & & 1.49 (1.10 to 2.01$)$ & 0.010 \\
\hline Fried food & & & & $<0.001$ & & \\
\hline Occasional & 9130 & $231(86.5)$ & $8899(93.0)$ & & Reference & \\
\hline Regular & 708 & $36(13.5)$ & $672(7.0)$ & & 1.71 (1.15 to 2.54$)$ & 0.008 \\
\hline Smoked food & & & & 0.064 & & \\
\hline Occasional & 9251 & $244(91.4)$ & 9007 (94.1) & & & \\
\hline Regular & 587 & $23(8.6)$ & $564(5.9)$ & & & \\
\hline Barbecue food & & & & 0.663 & & \\
\hline Occasional & 9307 & $251(94.0)$ & 9056 (94.6) & & & \\
\hline
\end{tabular}


Table 1 Continued

\begin{tabular}{|c|c|c|c|c|c|c|}
\hline Variable & $\begin{array}{l}\text { Total cohort } \\
(n=9838) \text { No. }\end{array}$ & $\begin{array}{l}\text { Gastric cancer } \\
(n=267) \text { No. }(\%)\end{array}$ & $\begin{array}{l}\text { Non-gastric cancer } \\
(n=9571) \text { No. }(\%)\end{array}$ & P value* & $\begin{array}{l}\text { Adjusted OR } \\
(95 \% \mathrm{Cl}) \dagger\end{array}$ & $P$ value \\
\hline Regular & 531 & $16(6.0)$ & $515(5.4)$ & & & \\
\hline Overnight leftovers & & & & 0.202 & & \\
\hline Occasional & 7083 & $183(68.5)$ & $6900(72.1)$ & & & \\
\hline Regular & 2755 & $84(31.5)$ & 2671 (27.9) & & & \\
\hline Red meat§ & & & & 0.806 & & \\
\hline Occasional & 4533 & $125(46.8)$ & $4408(46.1)$ & & & \\
\hline Regular & 5305 & $142(53.2)$ & $5163(53.9)$ & & & \\
\hline White meat§ & & & & 0.593 & & \\
\hline Occasional & 5517 & $154(57.7)$ & $5363(56.0)$ & & & \\
\hline Regular & 4321 & $113(42.3)$ & $4208(44.0)$ & & & \\
\hline Green vegetables & & & & 0.038 & & \\
\hline Occasional & 2144 & $72(27.0)$ & 2072 (21.6) & & & \\
\hline Regular & 7694 & $195(73.0)$ & 7499 (78.4) & & & \\
\hline Fresh fruits & & & & 0.009 & & \\
\hline Occasional & 4368 & $147(55.1)$ & 4491 (46.9) & & & \\
\hline Regular & 5200 & $120(44.9)$ & $5080(53.1)$ & & & \\
\hline
\end{tabular}

Data are presented as $\mathrm{n}(\%)$ for categorical variables, mean (SD) for continuous variables. For variables about eating habits, two categories for frequency of consumption were provided, that is, occasional (<3 times/week) and regular (at least three times/week).

${ }^{*} \mathrm{P}$ values refer to comparison between gastric cancer and non-gastric cancer groups in the univariate analysis.

†For variables not significant $(p>0.05)$ in the logistic regression model, multivariable data are not shown.

$\ddagger$ Body mass index: weight $(\mathrm{kg}) /$ height $(\mathrm{m})^{2}$.

$\S$ Red meat includes beef, pork and lamb. White meat includes fish, chicken and duck.

EIU, enzyme-immunoassay unit; G-17, gastrin-17; H. pylori, Helicobacter pylori; PG, pepsinogen.

prediction rule can be employed as an initial prescreening tool to identify individuals at an increased risk of GC (ie, individuals categorised by the prediction rule as having medium or high risk) for gastroscopy, which is a confirmatory method. Such a

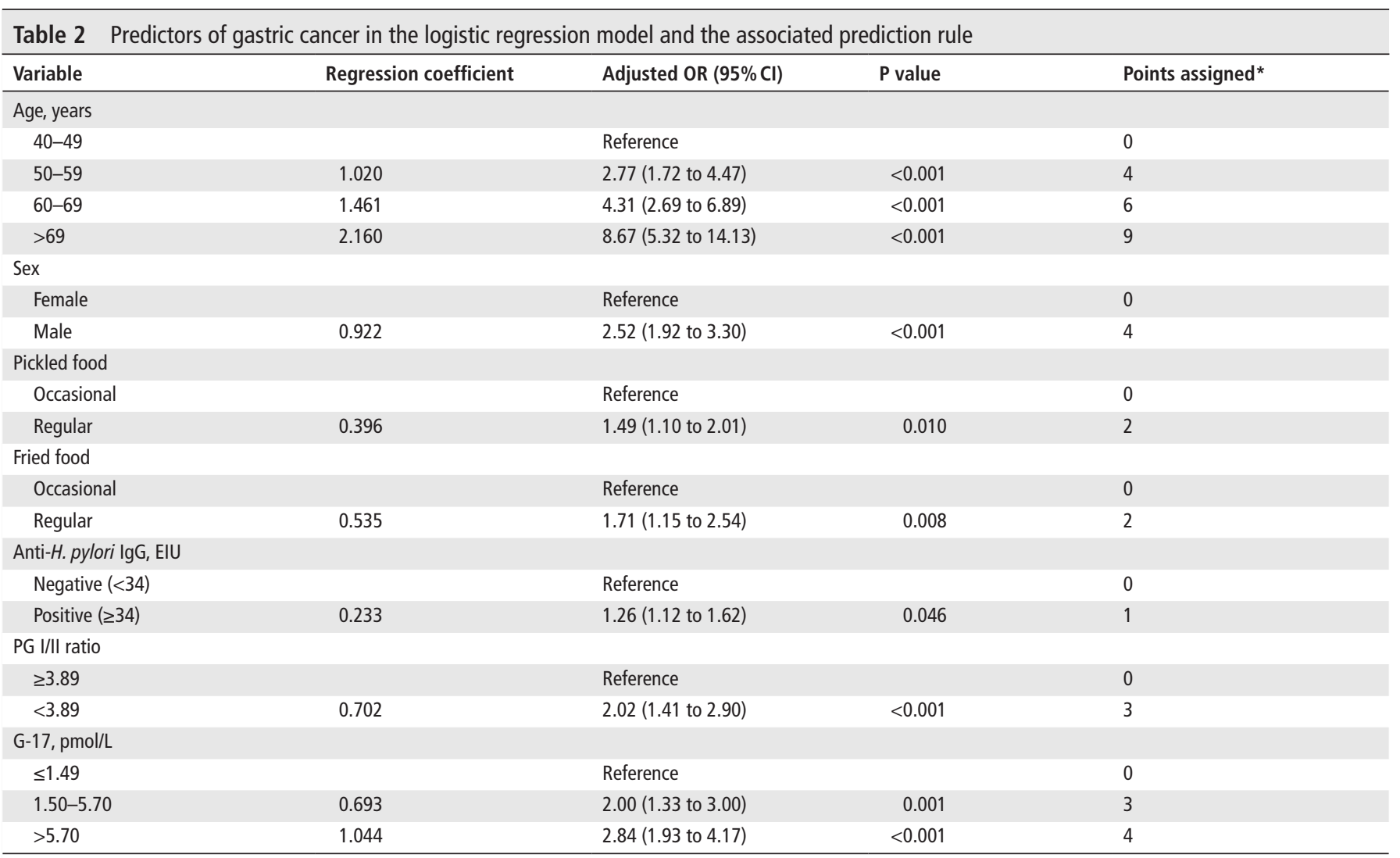

*Points were assigned by dividing the regression coefficients by the absolute value of the smallest coefficient in the model and rounding up to the nearest integer. A regression coefficient of 0.233 corresponded approximately to one point.

EIU, enzyme-immunoassay unit; G-17, gastrin-17; H. pylori, Helicobacter pylori; PG, pepsinogen. 
Table 3 Performance indices for the clinical prediction rule

\begin{tabular}{|c|c|c|c|c|}
\hline \multirow[b]{2}{*}{ Performance index } & \multirow{2}{*}{$\begin{array}{l}\text { Derivation cohort } \\
(\mathrm{n}=9838)\end{array}$} & \multirow{2}{*}{$\begin{array}{l}\text { Validation cohort } \\
(\mathrm{n}=5091)\end{array}$} & \multicolumn{2}{|c|}{ Internal validation* } \\
\hline & & & Mean (SD) & $95 \% \mathrm{Cl}$ \\
\hline$R^{2}$ & 0.024 & 0.018 & $0.025(0.002)$ & 0.024 to 0.025 \\
\hline Brier scores & 0.026 & 0.026 & $0.026(0.001)$ & 0.025 to 0.026 \\
\hline AUC & $0.76(0.73$ to 0.79$) \dagger$ & $0.73(0.68$ to 0.77$) \dagger$ & $0.76(0.01)$ & 0.71 to 0.80 \\
\hline Discrimination slope & 0.033 & 0.029 & $0.034(0.004)$ & 0.033 to 0.035 \\
\hline Calibration ( $p$ value) & 0.605 & 0.311 & $0.224(0.208)$ & 0.100 to 0.995 \\
\hline Calibration in the large ( $p$ value) & $<0.001$ & $<0.001$ & $0.028(0.005)$ & 0.024 to 0.032 \\
\hline Sensitivity (\%) & 70.8 & 69.6 & $70.7(2.7)$ & 65.5 to 76.1 \\
\hline Specificity (\%) & 67.8 & 66.8 & $67.8(0.5)$ & 66.9 to 68.8 \\
\hline Accuracy rate (\%) & 67.9 & 66.9 & $67.8(0.5)$ & 67.0 to 68.8 \\
\hline Positive predictive value (\%) & 5.8 & 5.5 & $5.8(0.4)$ & 5.0 to 6.7 \\
\hline Negative predictive value (\%) & 98.8 & 98.7 & $98.8(0.1)$ & 98.6 to 99.1 \\
\hline Positive likelihood ratio & 2.20 & 2.10 & $2.19(0.09)$ & 2.02 to 2.37 \\
\hline Negative likelihood ratio & 0.43 & 0.46 & $0.43(0.04)$ & 0.35 to 0.51 \\
\hline
\end{tabular}

*1000bootstrap samples were generated by using resampling with replacement, and averages of these samples were presented to demonstrate the validity of the prediction rule.

tAUC $(95 \% \mathrm{Cl})$.

$\mathrm{AUC}$, area under the receiver operating characteristic curve.

two-stage screening programme could identify approximately $70 \%$ of individuals with GC in the Chinese high-risk population. More importantly, one-third of individuals detected by the prediction rule had early-stage GC, and these cases accounted for over $60 \%$ of all cases with early GC in both the derivation and validation cohorts, indicating that the prediction rule has the potential to prescreening GC in early stage while greatly reducing the costs associated with gastroscopy. However, the rule did not screen well for gastric diseases other than GC (online supplementary table 6).

A thorough understanding of the risk factors for GC is important for identifying individuals at high risk who may need screening. In the present study, multivariable analysis showed that age, sex, serum PG I/II ratio, serum G-17 concentration, anti-H. pylori IgG status, consumption of pickled food and consumption of fried food were independent predictors for GC (table 1), and these were consistent with factors identified in previous studies. ${ }^{2}{ }^{18-20}$ There is abundant evidence that the incidence of GC increases with age, especially after 40 years old. ${ }^{6} 1820$ Compared with women, men have a higher risk of GC. ${ }^{18-20}$ The reasons for such differences include environmental or occupational exposures and physiological differences. ${ }^{19}$ 21-23 Pickled food and fried food, which are rich in $\mathrm{N}$-nitroso compounds, may increase the risk and incidence of GC. ${ }^{24}$ In addition, our data also indicate that the serum biomarkers (ie, PG I/PG II ratio and G-17) are valuable for the prescreening of non-junctional GC but not for junctional GC, as well as for intestinal-type GC but not diffuse-type GC (online supplementary tables 2-5).

H. pylori infection is considered to be the most prominent risk factor for GC. ${ }^{20}$ Since the discovery of $H$. pylori, numerous studies have associated it with GC and its precursors. ${ }^{2} 5182025$ The present study showed that $H$. pylori infection was weakly correlated with GC (table 1). This finding can be explained by the high prevalence of $H$. pylori-related preneoplastic conditions, including intraepithelial neoplasia, and atrophic gastritis, among our study participants. In the present study, the participants were only divided into GC and non-GC groups, and all individuals with these precancerous conditions were assigned to the non-GC group. Thus, the high prevalence of $H$. pylori-related precancerous conditions may have decreased the association between $H$. pylori infection and GC in the present study.

Two isoforms of PG, PG I and PG II, are present in serum. ${ }^{26}$ PG I is secreted mainly by chief cells in the fundic glands, while PG II is secreted by cells of the entire stomach and duodenum. ${ }^{2027} 28$ The serum PG I level decreases with loss of fundic gland mucosa. The serum PG II level remains relatively constant, although secretion may vary slightly in different upper gastrointestinal diseases. ${ }^{202728}$ Therefore, a low PG I level, a low PG I/II ratio or both is a good predictor for atrophic gastritis in the corpus. ${ }^{578} \mathrm{It}$ has been demonstrated that a low PG I/II ratio is strongly associated with the presence of GC, whereas PG I per se is not a significant predictor independent of the PG I/II ratio. ${ }^{5}$ In this study, a low PG I/II ratio was a strong predictor for a higher GC risk despite a high prevalence of atrophic gastritis. Indeed, previous studies also shown that a low PG I/II ratio is associated with a higher risk of developing GC. ${ }^{5} 7$

The serum G-17 level depends on intragastric acidity and the number of $\mathrm{G}$ cells in the antrum. ${ }^{20}$ Atrophic gastritis in different topographic locations may result in decreased, normal or increased levels of G-17. ${ }^{18}$ The present study showed that individuals with GC had a higher serum level of G-17 than those without, which is consistent with previous studies concluding that an individual with a higher serum G-17 level has a higher risk of GC. ${ }^{82}$ It has been proposed that G-17 has a proliferative effect on gastric mucosa and may directly promote gastric carcinogenesis. ${ }^{30} 31$ Moreover, it has been reported that there is a link between body dominant gastritis and atrophy as a key risk condition, which in turn leads to an increased G17 response from the antrum. ${ }^{32}$ It should be emphasised that G-17 is generally considered instable and thus not commonly included in prediction models; however, it was included in the five biomarker-based method. ${ }^{5}$ In the present study, serum samples were required to be stored at room temperature $\left(\leq 25^{\circ} \mathrm{C}\right)$ and immediately assayed within 3 hours. The results showed that the AUCs of the models with G-17 were higher than those of the responding models without G-17 in both the derivation and validation cohorts (online supplementary tables 7-9). Therefore, we recommend including G-17 in the prediction models for accurate risk stratification in prescreening for GC prior to gastroscopy if the serum samples are properly prepared, stored and tested within 3 hours. 
Table 4 Risk of gastric cancer by risk score and risk category

\begin{tabular}{|c|c|c|c|c|c|c|}
\hline Risk score & Total number & $\begin{array}{l}\text { Gastric cancer* } \\
\text { No. }(\%)\end{array}$ & Risk category† & $\begin{array}{l}\text { Total number } \neq \\
\text { No. }(\%)\end{array}$ & $\begin{array}{l}\text { Gastric cancer* } \\
\text { No. }(\%)\end{array}$ & $\begin{array}{l}\text { Number needed to } \\
\text { screen§ }\end{array}$ \\
\hline Derivation cohort & & & & $9838(100.0)$ & $267(2.7)$ & 37 \\
\hline 0 & 206 & $0(0.0)$ & Low (0-11) & $6564(66.7)$ & $78(1.2)$ & 84 \\
\hline 1 & 107 & $0(0.0)$ & & & & \\
\hline 2 & 51 & $0(0.0)$ & & & & \\
\hline 3 & 248 & $0(0.0)$ & & & & \\
\hline 4 & 857 & $7(0.8)$ & & & & \\
\hline 5 & 491 & $5(1.0)$ & & & & \\
\hline 6 & 345 & $1(0.3)$ & & & & \\
\hline 7 & 639 & $4(0.6)$ & & & & \\
\hline 8 & 1099 & $12(1.1)$ & & & & \\
\hline 9 & 917 & $13(1.4)$ & & & & \\
\hline 10 & 832 & $20(2.4)$ & & & & \\
\hline 11 & 772 & $16(2.1)$ & & & & \\
\hline 12 & 687 & $24(3.5)$ & Medium (12-16) & $2715(27.6)$ & $120(4.4)$ & 23 \\
\hline 13 & 775 & $26(3.4)$ & & & & \\
\hline 14 & 622 & $31(5.0)$ & & & & \\
\hline 15 & 367 & $19(5.2)$ & & & & \\
\hline 16 & 264 & $20(7.6)$ & & & & \\
\hline 17 & 283 & $28(9.9)$ & High $(>16)$ & $559(5.7)$ & $69(12.3)$ & 8 \\
\hline 18 & 141 & $18(12.8)$ & & & & \\
\hline 19 & 52 & $6(11.5)$ & & & & \\
\hline 20 & 49 & $12(24.5)$ & & & & \\
\hline 21 & 25 & $4(16.0)$ & & & & \\
\hline 22 & 8 & $1(12.5)$ & & & & \\
\hline 24 & 1 & $0(0.0)$ & & & & \\
\hline Validation cohort & & & & $5091(100.0)$ & $138(2.7)$ & 37 \\
\hline 0 & 123 & $1(0.8)$ & Low (0-11) & $3352(65.8)$ & $42(1.3)$ & 80 \\
\hline 1 & 46 & $0(0.0)$ & & & & \\
\hline 2 & 19 & $0(0.0)$ & & & & \\
\hline 3 & 133 & $1(0.8)$ & & & & \\
\hline 4 & 424 & $3(0.7)$ & & & & \\
\hline 5 & 256 & $2(0.8)$ & & & & \\
\hline 6 & 184 & $2(1.1)$ & & & & \\
\hline 7 & 302 & $3(1.0)$ & & & & \\
\hline 8 & 532 & $12(2.3)$ & & & & \\
\hline 9 & 512 & $7(1.4)$ & & & & \\
\hline 10 & 429 & $4(0.9)$ & & & & \\
\hline 11 & 392 & $7(1.8)$ & & & & \\
\hline 12 & 376 & $15(4.0)$ & Medium (12-16) & $1460(28.7)$ & $65(4.5)$ & 22 \\
\hline 13 & 443 & $11(2.5)$ & & & & \\
\hline 14 & 317 & $17(5.4)$ & & & & \\
\hline 15 & 179 & $10(5.6)$ & & & & \\
\hline 16 & 145 & $12(8.3)$ & & & & \\
\hline 17 & 130 & $16(12.3)$ & High $(>16)$ & $279(5.5)$ & $31(11.1)$ & 9 \\
\hline 18 & 76 & $5(6.6)$ & & & & \\
\hline 19 & 32 & $2(6.3)$ & & & & \\
\hline 20 & 22 & 3 (13.6) & & & & \\
\hline 21 & 12 & $4(33.3)$ & & & & \\
\hline 22 & 4 & $1(25.0)$ & & & & \\
\hline 24 & 3 & $0(0.0)$ & & & & \\
\hline
\end{tabular}

*The prevalence rate of gastric cancer.

tIn both derivation and validation cohorts, there is a significant difference in prevalence of gastric cancer across the three risk categories ( $p<0.001)$.

$\ddagger$ Proportion relative to all participants of the derivation or validation cohort.

$\S$ Defined as the number of participants who should undergo gastroscopy screening to identify one patient with gastric cancer.

A positive family history of GC among first-degree relatives is known to be a risk factor for GC. ${ }^{33} 34$ According to the progression model for the intestinal type of GC proposed by Correa ${ }^{35}$ and previous studies, a positive family history of 
Table 5 Comparison between the prediction rule and alternative prediction methods in the validation cohort

\begin{tabular}{|c|c|c|c|c|}
\hline Prediction method & Predictors & Score range or risk groups & AUC $(95 \% \mathrm{Cl})$ & $P$ value \\
\hline This study & $\begin{array}{l}\text { Age, sex, H. pylori infection, PG I/ } \\
\text { II ratio, G-17, pickled food, and } \\
\text { fried food. }\end{array}$ & 0 to 25 & 0.757 (0.729 to 0.786$)$ & Reference \\
\hline ABC method by Miki ${ }^{9 *}$ & $\begin{array}{l}\text { H. pylori infection, PG I and PG I/ } \\
\text { II ratio. }\end{array}$ & Groups A, B, C and D & 0.527 (0.476 to 0.579$)$ & $<0.001$ \\
\hline $\begin{array}{l}\text { ELISA-based ABC method in } \\
\text { China }{ }^{56}+\end{array}$ & $\begin{array}{l}\text { H. pylori infection, PG I and PG I/ } \\
\text { II ratio. }\end{array}$ & Groups A, B, C and D & 0.575 (0.522 to 0.629$)$ & $<0.001$ \\
\hline $\begin{array}{l}\text { Five markers-based method by } \\
\text { Tu et } a l^{5}\end{array}$ & $\begin{array}{l}\text { H. pylori infection, PG I, PG II, PG } \\
\text { I/II ratio and G-17. }\end{array}$ & 0 to 21 & 0.589 (0.548 to 0.630$)$ & $<0.001$ \\
\hline
\end{tabular}

*Subjects were classified into 1 of 4 risk groups based on the results of the two serologic tests, anti-H. pylori lgG antibody titres and the PG I and II levels: group A [H. pylori(-) and PG(-)], group B $[$ H. pylori(+) and PG(-)], group C $[$ H. pylori(+) and PG(+)] and group D $[$ H. pylori (-) and PG(+)]. For the PG method, the cut-off points for identifying the risk of gastric cancer should be $\leq 70 \mathrm{ng} / \mathrm{mL}$ for $\mathrm{PG}$ I and $\leq 3.0$ for the $\mathrm{PGI} / \mathrm{Il}$ ratio.

tThe risk stratification method was the same as the $A B C$ method by Miki except the definition of abnormal PG, that is, $\leq 70 \mathrm{ng} / \mathrm{mL}$ for $P G$ I and $\leq 7.0$ for the PGI/II ratio. AUC, area under the receiver operating characteristic curve; ELISA, enzyme-linked immunosorbent assay; G-17, gastrin-17; H. pylori, Helicobacter pylori; PG, pepsinogen.

GC is also a risk factor for precancerous gastric conditions, including gastric atrophy. ${ }^{36}$ This may explain why a positive family history was not sufficient to distinguish the GC group from the non-GC group in the present study. The same also applies to smoking. Several meta-analyses have suggested that smoking is a risk factor for GC, ${ }^{37-40}$ as well as for precancerous gastric conditions. ${ }^{4142}$

In the present study, almost all known key risk factors for GC were included in the analysis, and the new prediction rule developed from the analysis produced good performance metrics (table 3), which were both internally and externally validated. Therefore, the prediction rule is believed to be robust and valid, and thus, have generalisable prescreening performance in the Chinese 'high-risk' population.

In addition, the prediction rule developed in the present study showed the best discrimination among the four prediction methods as assessed in the validation cohort (table 5). There are several explanations for the poor discriminative ability of the other three methods. First, none of the alternative methods ${ }^{569}$ include age and sex, which are strong predictors for GC. Second, H. pylori infection is a risk factor for GC precancerous gastric conditions. Therefore, the ABC methods combining $H$. pylori infection and PG obviously did not show good discrimination in our study population. Third, the PG cut-off values in the $\mathrm{ABC}$ methods were used for determining atrophic gastritis rather than GC, and thus did not show good performance for GC. Finally, because the PG I/II ratio is not independent of PG I and PG II, it is not appropriate to use a logistic regression method including all three variables to develop the five biomarker-based method. ${ }^{5}$

It is noticed that the prediction rule performed well for non-junctional GC and intestinal-type GC, but not for junctional GC and diffuse-type GC. In a recent study, Bornschein et al categorised adenocarcinomas at the gastro-oesophageal junction into GOJ1 (1-5 cm proximal to the junction, also distal oesophageal), GOJ2 ('true' junctional), and GOJ3 $(2-5 \mathrm{~cm}$ distal to the junction, also proximal gastric) and examined the association of with preneoplastic conditions and inflammation of the gastric mucosa. ${ }^{44}$ They found that whereas GOJ1 cancers, which are of esophageal nature, were almost exclusively (98.4\%) of the intestinal type, GOJ2 and GOJ3, which are of gastric nature, were the intestinal type in $77.4 \%$ and $77.6 \%$ of the cases. In the present study, intestinal-type GC was present in $88.9 \%$ (40/45) of junctional cancer and 61.1\% (220/360) of non-junctional GC (online supplementary table 10). It has been generally accepted that, although junctional GC and non-junctional
GC share several demographic and environmental risk factors, obesity and GORD are exclusive risk factors for junctional GC, whereas $H$. pylori infection and high intake of salty and smoked foods are exclusive risk factors for non-junctional GC, ${ }^{18}$ which explains why the prediction rule performs better for non-junctional GC than for junctional GC. In addition, it is well established that the development of intestinal-type GC, specifically in non-junctional locations, follows Correa's cascade, ${ }^{35}$ and H. pylori infection plays a critical role in initiating acute and chronic gastric inflammation, which may progress into gastric atrophy associated with changes in the expression of PG and G17. Therefore, it is conceivable that a predictive rule based on these factors would achieve better performance for prescreening intestinal-type GC than for diffuse-type GC.

Notably, although the prediction rule developed in the present study has good discrimination with an AUC of 0.76 , high sensitivity (70.8\%), good calibration (0.629) and obvious low cost (compared with gastroscopy for all of the high-risk population), the PPV was only $5.8 \%$ in the derivation cohort and $5.5 \%$ in the validation cohort. It should be kept in mind though that this study aimed to develop a prescreening tool not a diagnostic tool. In fact, screening methods for cancers developed in previous studies generally have low PPVs, for example, mammography $(3.2 \%-4.5 \%)$ for breast cancer, ${ }^{45} 46$ the faecal occult blood test $(5.2 \%-10.7 \%)^{47} 48$ and faecal immunochemical test $(7.3 \%-$ $12 \%)^{49} 50$ for colorectal cancer and prostate-specific antigen $(2.9 \%-3.6 \%)$ for prostate cancer. ${ }^{51}$ In the contrast, the current model owns a high NPV as $98.8 \%$, which means it can reduce unnecessary endoscopy screening.

It must be mentioned that approximately 30\% of GC cases in the population would be missed if the prediction model were applied for prescreening of GC despite the high NPV. Therefore, whereas the prediction rule is recommended as an initial prescreening tool for GC in a large population, healthcare providers should keep the above-mentioned point in mind and seriously consider all risk factors when they refer individuals for endoscopy, in order to avoid missing GC cases, especially those with junctional or diffuse-type GC.

There are several potential limitations in the present study. First, for practical purposes, the protocol for taking biopsy specimens in the present study was different from that proposed in the updated Sydney system. Thus, the operative link on gastritis assessment and operative link on gastritis assessment on intestinal metaplasia systems, which have been increasingly used to assess the gastric preneoplastic conditions and GC in the last decade, ${ }^{52-54}$ were not included 
in the present study. Second, early GC was only diagnosed from the biopsy specimens, which may be a little confused with intraepithelial neoplasia and thus decreased the diagnosis accuracy. Third, we did not collect data on central adiposity, which is reported to be associated with GC risk. Thus, inclusion of central adiposity, instead of body mass index, would be more appropriate in the analysis. We will keep this important point in mind and incorporate it into our future study. Fourth, there were some GC cases complicated with other gastric diseases, which maybe of impacts on the levels of serum biomarkers, and thus affect the application of the prediction rule. However, our data showed that GC cases with other gastric diseases were more likely to be identified with the prediction rule and subjected to endoscopy compared with GC alone, in despite of no statistical difference (online supplementary table 11). Finally, a data management platform was used to collect all data, and only the participants for whom all data were successfully entered into the platform were included in the analysis; thus, those with any missing data were automatically excluded from the analysis, which may have resulted in some bias.

In conclusion, the prediction rule developed and validated in the present study has good performance in identifying individuals at higher risk of GC prior to gastroscopy among the Chinese population. Thus, it can be used as an accurate and cost-effective initial mass-prescreening tool to improve the detection of GC, including early GC, thus improving the secondary prevention of GC. A future large population-based screening project should be launched to test its feasibility.

\section{Author affiliations}

'Department of Gastroenterology, Changhai Hospital, Naval Medical University, Shanghai, China

${ }^{2}$ Tumor Etiology and Screening Department, China Medical University, Shenyang, China

${ }^{3}$ Department of Gastroenterology, Mianyang Central Hospital, Mianyang, China ${ }^{4}$ Department of Gastroenterology, Affiliated Hospital of Yan'an University, Yanan, China

${ }^{5}$ Department of Gastroenterology, Baoding First Central Hospital, Baoding, China ${ }^{6}$ Department of Gastroenterology, Baoji Central Hospital, Baoji, China

${ }^{7}$ Department of Gastroenterology, Anhui Provincial Hospital, Hefei, China

${ }^{8}$ Department of Gastroenterology, Affiliated Hospital of Ningbo University, Ningbo,

China

${ }^{9}$ Department of Gastroenterology, Taizhou Hospital, Taizhou, China

${ }^{10}$ Department of Gastroenterology, First Affiliated Hospital of Nanchang University, NanChang, China

${ }^{11}$ Department of Gastroenterology, Beijing Traditional Chinese Medicine Hospital of Capital Medical University, Beijing, China

${ }^{12}$ Department of Gastroenterology, Affiliated Hospital of Binzhou Medical College, Binzhou, China

${ }^{13}$ Department of Gastroenterology, Yan'an People's Hospital, Yan'an, China

${ }^{14}$ Department of Gastroenterology, Affiliated Hospital of Guizhou Medical University, Guiyang, China

${ }^{15}$ Department of Gastroenterology, Ruian People's Hospital, Rui'an, China ${ }^{16}$ Department of Gastroenterology, Wenzhou Central Hospital, Wenzhou, China

${ }^{17}$ Department of Gastroenterology, Ankang Central Hospital, Ankang, China

${ }^{18}$ Department of Gastroenterology, Taizhou Municipal Hospital, Taizhou, China

${ }^{19}$ Department of Gastroenterology, Zhejiang General Team Hospital of Chinese

People's Armed Police Force, Hangzhou, China

${ }^{20}$ Department of Gastroenterology, Ningbo Yinzhou District Second Hospital, Ningbo, China

${ }^{21}$ Department of Gastroenterology, Anqing First People's Hospital, Anqing, China

${ }^{22}$ Department of Gastroenterology, Second Affiliated Hospital of South China University, Hengyang, China

${ }^{23}$ Department of Gastroenterology, Henan Provincial People's Hospital, Zhengzhou, China

${ }^{24}$ Department of Gastroenterology, Shanghai Songjiang Distict Central Hospital, Shanghai, China

${ }^{25}$ Department of Gastroenterology, Ningbo Beilun District Xiaogang Hospital, Ningbo, China

${ }^{26}$ Department of Gastroenterology, Binzhou People's Hospital, Binzhou, China
${ }^{27}$ Department of Gastroenterology, Jianyang People's Hospital, Jianyang, China

Correction notice This article has been corrected since it published Online First. The acknowledgements section has been updated.

Acknowledgements We would like to thank Yuling Wang, Qianqian Zhu, Guixiang Li, Wei Zhu and Pingping Zhang, Changhai Hospital of Naval Medical University, Shanghai, for assistance in controlling the quality of the data, and Jianming Zheng, Changhai Hospital of Naval Medical University, Shanghai, for final histological diagnosis. We would also like to thank the following other members of the Chinese Gastric Cancer Study Group for enrolling their patients in this study: Dongmei Xiang, Mianyang Central Hospital, Mianyang; Donghong Yuan, Affiliated Hospital of Yan'an University, Yan'an; Yiqian Huang, Baoding First Central Hospital, Baoding; Yujiang Wu, Baoji Central Hospital, Baoji; Qiaomin Wang, Anhui Provincial Hospital, Hefei; Kefeng Hu, Affiliated Hospital of Ningbo University, Ningbo; Xinli Mao and Chao Wang, Taizhou Hospital, Taizhou; Xu Shu, First Affiliated Hospital of Nanchang University, Nanchang; Zhengguang Du, Beijing Traditional Chinese Medicine Hospital of Capital Medical University, Beijing; Kun Li, Affiliated Hospital of Binzhou Medical College, Binzhou; Jiankui Wang, Yan'an People's Hospital, Yan'an; Dongping Zhang, Affiliated Hospital of Guizhou Medical University, Guiyang; Gengyu Li, Ruian People's Hospital, Ruian; Yibing Zhang, Wenzhou Central Hospital, Wenzhou; Changqin Luo, Ankang Central Hospital, Ankang; Feifei Mo, Taizhou Municipal Hospital, Taizhou; Shengzhi Xu, Zhejiang General Team Hospital of the Chinese People's Armed Police Force, Hangzhou; Qi Lin, Ningbo Yinzhou District Second Hospital, Ningbo; Fanglai Zhu, Anqing First People's Hospital, Anqing; Guoqin Li, Second Affiliated Hospital of South China University, Hengyang; Manru Ma, Henan Provincial People's Hospital, Zhengzhou; Yan Qiu, Shanghai Songjiang Distict Central Hospital, Shanghai; Lina Xie, Ningbo Beilun District Xiaogang Hospital, Ningbo; Jun Wang, Binzhou People's Hospital, Binzhou; Jinwen Liao, Jianyang People's Hospital, Jianyang; Jie Zhang and Zhen Yan, Beijing Anzhen Hospital of Capital Medical University, Beijing; Haifan Yan, Wenling First People's Hospital, Wenling; Xingxiang He and Wenrui Xie, The First Affiliated Hospital of Guangdong Pharmacy College, Guangzhou; Bin Lv and Haifeng Jin, Zhejiang Provincial Traditional Chinese Medicine Hospital, Hangzhou; Junping Wang and Yunfeng Yan, Shanxi Provincial People's Hospital, Taiyuan; Yikuan Feng and Linjun Mou, Weifang People's Hospital, Weifang; Yuanqing He and Wenping Zhou, Guangyuan Third People's Hospital, Guangyuan; Wen Wang and Zhou Ye, Fuzhou Military District General Hospital, Fuzhou; Shibing Feng and Bai Wang, Beijing Hepingli Hospital, Beijing; Qiang Guo and Zan Zuo, First People's Hospital of Yunan Province, Kunming; Jianxiang Wang, Chengdu Fifth People's Hospital, Chengdu; Li Cui and Jiujun Wang, Beijing Yanhua Hospital, Beijing; Yi Jian and Wu Wen, Chengdu Second People's Hospital, Chengdu; Xiuyin Ye and Guoping Du, Foshan Shunde District First People's Hospital, Foshan; Minzhi Li and Liuping Jia, Foshan Nanhai District People's Hospital, Foshan; Lei Wang and Qiang Zhan, Wuxi People's Hospital, Wuxi; Bing Hu and Ersun Jiang, West China Hospital, Chengdu; Jing Hu and Peilin Li, Fuxing Hospital of Capital Medical University, Beijing; Xiaogang Liu and Lianping Li, Sichuan Provincial People's Hospital, Chengdu; Aijun Liao and Hua Zhong, First Affiliated Hospital of South China University, Hengyang; Huaili Xu and Li Tian, Xidian Group Hospital, Xi'an; Dongyuan Xiu and Shiquan Wang, Chongzhou People's Hospital of Sichuan Province, Chongzhou; Jun Xue, Chifeng People's Hospital, Chifeng; Lijiang Huang and Hong Sheng, Ningbo Fourth People's Hospital, Ningbo; Xiaofeng Zhang and Jianfeng Yan, Hangzhou First People's Hospital, Hangzhou; Shaofeng Wang, Changzhi People's Hospital, Changzhi; Zhijian Chen, Wenzhou Traditional Chinese Medicine Hospital, Wenzhou; Guoxin Zhang and Yini Dang, Jiangsu Provincial People's Hospital, Nanjing; Jiguang Kou, Xiaogan Central Hospital, Xiaogan; Yu Lan and Cunzeng Jia, Beijing Jishuitan Hospital, Beijing; Lan Li, Guizhou Provincial People's Hospital, Guiyang; Xiaobin Sun, Chengdu Third People's Hospital, Chengdu; Yujie Liu, Mingzhou Hospital of Zhejiang University, Hangzhou; Liping Yi, Beijing Shijingshan Hospital, Beijing; Jie Zhang, Longyan Second Hospital, Longyan; Zuoyan Wu, Beijing Sixth Hospital, Beijing; Yana Huang, Yibin Second People's Hospital, Yibin; Lin Jia, Guangzhou Nansha Central Hospital, Guangzhou; Hong Ouyang, Lin'an People's Hospital, Lin'an; Jianming Xu, Affiliated Hospital of Anhui Medical University, Hefei; Zeli Gao, Shanghai Pudong New Area People's Hospital, Shanghai; Sujuan Fei, Affiliated Hospital of Xuzhou Medical College, Xuzhou; Jun Yao, Zhenjiang First People's Hospital, Zhenjiang; Jiancun Xiao, Beijing Coal Group General Hospital, Beijing; Xiaoyan Wang, Xiangya Third Hospital of Central South University, Changsha; Zhihua Zhang, Leshan Traditional Chinese Medicine Hospital, Leshan; Weidong Zhao, Shengli Oilfield Central Hospital of Binzhou Medical University, Dongying; Mingqing Zhang, 75th Hospital of Chinese People's Liberation Army, Zhangzhou; Jiandong Li, 285th Hospital of Chinese People's Liberation Army, Handan; Zhentao Li, Dengfeng People's Hospital, Dengfeng; Gaifan Liu, The Third Affiliated Hospital of Hebei Medical University, Shijiazhuang; Linglong Xue, Taigang Group General Hospital, Taiyuan; Delian Liu, Xiangya Second Hospital of Central South University, Changsha; Zhaohong Shi, Wuhan First Hospital, Wuhan; Haitao Zhang, Shanxi Aerospace Hospital, Xi'an; Maoling Zhu, Shanghai Yangpu District Central Hospital, Shanghai; Jin Wang, Coal General Hospital, Beijing; Yushan He, Shaoyang Central Hospital, Shaoyang; Bin Yang, Taizhou People's Hospital, Taizhou; Chunying Zhai, Beijing Puren Hospital, Beijing; Qing Wang, Linfen People's Hospital, Linfen; Bin Xu, Huainan People's Hospital, Huainan; Feng Gao, 
People's Hospital of Xinjiang Uygur Automous Region, Wulumuqi; Qi Wang, Second Hospital of Shanxi Medical University, Taiyuan; Yan Shi, Bethune First Hospital of Jilin University, Changchun; Jinkang Xu, Kunshan Traditional Chinese Medicine Hospital, Kunshan; Hongqing Qiu, Zhangjiagang First People's Hospital, Zhangjiagang; Jianlin Ren, Zhongshan Hospital of Xiamen University, Xiamen; Cheng Zhang, Zhoupu Hospital, Shanghai; Yihai Shi, Gongli Hospital of Second Military University, Shanghai; Liping He, Fujian Provincial Hospital, Fuzhou; Guoqi Zheng, Cangzhou Central Hospital, Cangzhou; Xiaojun Huang, Second Hospital of Lanzhou University, Lanzhou; Weishun Chen, Zhuzhou Central Hospital, Zhuzhou; Guikai Wu, Tangshan Worker's Hospital, Tangshan; Dongxu Wang, 254th Hospital of Chinese People's Liberation Army, Tianjin; Peng Yan, Liaocheng People's Hospital, Liaocheng; Qing'e Liu, Handan Central Hospital, Handan; Shihai Xia, Affiliated Hospital of Chinese People's Armed Police Force Logistics Academy, Tianjin; Baochun Hu, Armed Police Guangdong General Hospital, Guangzhou; Jinkun Zhang, Suzhou Municipal Hospital, Suzhou; Yongning Zhou, First Hospital of Lanzhou University, Lanzhou; Fangxin Zhang, Lanzhou Military Region General Hospital, Lanzhou; Libing He, Wuan First People's Hospital, Wuan; Hong Deng, Deyang People's Hospital, Deyang; Shaoneng Liu, Guang'anmen Hospital of China Academy of Chinese Medical Sciences, Beijing; Jianhua He, Central Hospital of Enshi Tujia and Miao Autonomous Prefecture, Enshi; Qi Zhao, Second Affiliated Hospital of Guiyang College of Traditional Chinese Medicine, Guiyang; and Xiangshuan Ge, Hongli Hospital of He'nan Province, ChangYuan, China. We also thank Medjaden Bioscience Limited for providing assistance in preparing the manuscript.

Contributors Conception and design: Q-C, YY, YD and ZL; financial support: YD and ZL; administrative support: Q-C and CZ; provision of study materials or patients: $Q F, Y F, Y H, J L, K Z, G Y, L Y, N L, S Z, C L, M L, Q L, R L, J P, X Y, X Z, Y L, B L, A L, H C, X L, P X, J Z$, $\mathrm{BL}$ and ZD; collection and assembly of data: Q-CC, CZ, QF, YF, YH, JL, KZ, GY, LY, NL, $S Z, C L, M L, Q L, R L, J P, X Y, X Z, Y L, B L, A L, H C, X L, P X, J Z, B L$ and $Z D$.

Funding This work was supported by the National Science and Technology Plan Project of the Ministry of Science and Technology of China (Grant No. 2015BAl13B08).

Competing interests None declared.

Patient consent for publication Not required.

Ethics approval The study protocol was approved by the ethics committees of the respective institutions (the leading approval as CHEC2014-066 by Changhai Hospital).

Provenance and peer review Not commissioned; externally peer reviewed.

Open access This is an open access article distributed in accordance with the Creative Commons Attribution Non Commercial (CC BY-NC 4.0) license, which permits others to distribute, remix, adapt, build upon this work non-commercially, and license their derivative works on different terms, provided the original work is properly cited, appropriate credit is given, any changes made indicated, and the use is non-commercial. See: http://creativecommons.org/licenses/by-nc/4.0/.

\section{REFERENCES}

1 Chen W, Zheng R, Baade PD, et al. Cancer statistics in China, 2015. CA Cancer J Clin 2016;66:115-32.

2 Nie $Y$, Wu K, Yu J, et al. A global burden of gastric cancer: the major impact of China. Expert Rev Gastroenterol Hepatol 2017;11:651-61.

3 Zong L, Abe M, Seto Y, et al. The challenge of screening for early gastric cancer in China. Lancet 2016;388:2606

4 Leja M, You W, Camargo MC, et al. Implementation of gastric cancer screening - the global experience. Best Pract Res Clin Gastroenterol 2014;28:1093-106.

5 Tu H, Sun L, Dong X, et al. A Serological Biopsy Using Five Stomach-Specific Circulating Biomarkers for Gastric Cancer Risk Assessment: A Multi-Phase Study. Am J Gastroenterol 2017;112:704-15.

6 Chinese Society of Digestive Endoscopy. Consensus on screening and endoscopic diagnosis and treatment of early gastric cancer in China (Changsha, 2014). Zhonghua Xiao Hua Nei Jing Za Zhi 2014;31:361-77.

7 Watabe H, Mitsushima T, Yamaji Y, et al. Predicting the development of gastric cancer from combining Helicobacter pylori antibodies and serum pepsinogen status: a prospective endoscopic cohort study. Gut 2005;54:764-8.

8 Kurilovich S, Belkovets A, Reshetnikov 0, et al. Stomach-specific Biomarkers (GastroPanel) Can Predict the Development of Gastric Cancer in a Caucasian Population: A Longitudinal Nested Case-Control Study in Siberia. Anticancer Res 2016;36:247-53.

9 Miki K. Gastric cancer screening by combined assay for serum anti-Helicobacter pylori IgG antibody and serum pepsinogen levels - "ABC method". Proceedings of the Japan Academy, Series B 2011;87:405-14.

10 Gao D, Zhao J, Yu X, et al. Clinical values of gastrin 17 and pepsinogen in gastric cancer and precancerous lesion screening. Biomedical Research2017;28:6140-3.

11 Dixon MF, Genta RM, Yardley JH, et al. Classification and grading of gastritis. The updated Sydney System. International Workshop on the Histopathology of Gastritis, Houston 1994. Am J Surg Pathol 1996;20:1161-81.
12 Schlemper RJ, Riddell RH, Kato Y, et al. The Vienna classification of gastrointestinal epithelial neoplasia. Gut 2000;47:251-5.

13 Dixon MF. Gastrointestinal epithelial neoplasia: Vienna revisited. Gut 2002;51:130-1.

14 Cai QC, Yu ED, Xiao Y, et al. Derivation and validation of a prediction rule for estimating advanced colorectal neoplasm risk in average-risk Chinese. Am J Epidemiol 2012;175:584-93.

15 Moons KG, Harrell FE, Steyerberg EW. Should scoring rules be based on odds ratios or regression coefficients? J Clin Epidemiol 2002;55:1054-5.

16 Steyerberg EW, Harrell FE, Borsboom GJ, et al. Internal validation of predictive models: efficiency of some procedures for logistic regression analysis. J Clin Epidemiol 2001;54:774-81.

17 Steyerberg EW, Bleeker SE, Moll HA, et al. Internal and external validation of predictive models: a simulation study of bias and precision in small samples. I Clin Epidemiol 2003;56:441-7.

18 Karimi P, Islami F, Anandasabapathy S, et al. Gastric cancer: descriptive epidemiology, risk factors, screening, and prevention. Cancer Epidemiol Biomarkers Prev 2014;23:700-13.

19 González CA, Agudo A. Carcinogenesis, prevention and early detection of gastric cancer: where we are and where we should go. Int J Cancer2012;130:745-53.

20 Leung WK, Wu MS, Kakugawa Y, et al. Asia Pacific Working Group on Gastric Cancer. Screening for gastric cancer in Asia: current evidence and practice. Lancet Oncol 2008:9:279-87.

21 Derakhshan MH, Liptrot S, Paul J, et al. Oesophageal and gastric intestinal-type adenocarcinomas show the same male predominance due to a 17 year delayed development in females. Gut 2009;58:16-23.

22 Sipponen P, Correa P. Delayed rise in incidence of gastric cancer in females results in unique sex ratio (M/F) pattern: etiologic hypothesis. Gastric Cancer 2002;5:213-9.

23 Camargo MC, Goto Y, Zabaleta J, et al. Sex hormones, hormonal interventions, and gastric cancer risk: a meta-analysis. Cancer Epidemiol Biomarkers Prev 2012;21:20-38.

24 Wogan GN, Hecht SS, Felton JS, et al. Environmental and chemical carcinogenesis. Semin Cancer Biol 2004;14:473-86.

25 You WC, Zhang L, Gail MH, et al. Gastric dysplasia and gastric cancer: Helicobacter pylori, serum vitamin C, and other risk factors. J Nat/ Cancer Inst 2000;92:1607-12.

26 Pepsinogens SIM. pepsins, and pepsin inhibitors. Gastroenterology 1971;60:586-604.

27 Chiang TH, Chiu SY, Chen SL, et al. Serum Pepsinogen as a Predictor for Gastric Cancer Death: A 16-Year Community-based Cohort Study. J Clin Gastroenterol 2018.

28 Zagari RM, Rabitti S, Greenwood DC, et al. Systematic review with meta-analysis: diagnostic performance of the combination of pepsinogen, gastrin-17 and antiHelicobacter pylori antibodies serum assays for the diagnosis of atrophic gastritis. Aliment Pharmacol Ther 2017;46:657-67.

29 Sun L, Tu H, Liu J, et al. A comprehensive evaluation of fasting serum gastrin-17 as a predictor of diseased stomach in Chinese population. Scand I Gastroenterol 2014:49:1164-72.

30 Sawada M, Dickinson CJ. The G cell. Annu Rev Physiol 1997;59:273-98.

31 Wang TC, Dangler CA, Chen D, et al. Synergistic interaction between hypergastrinemia and Helicobacter infection in a mouse model of gastric cancer. Gastroenterology 2000;118:36-47

32 Haj-Sheykholeslami A, Rakhshani N, Amirzargar A, et al. Serum pepsinogen I, pepsinogen II, and gastrin 17 in relatives of gastric cancer patients: comparative study with type and severity of gastritis. Clin Gastroenterol Hepatol 2008;6:174-9.

33 Yaghoobi M, McNabb-Baltar J, Bijarchi R, et al. What is the quantitative risk of gastric cancer in the first-degree relatives of patients? A meta-analysis. World I Gastroenterol 2017;23:2435-42.

34 Bernini M, Barbi S, Roviello F, et al. Family history of gastric cancer: a correlation between epidemiologic findings and clinical data. Gastric Cancer 2006;9:9-13.

35 Correa P. Human gastric carcinogenesis: a multistep and multifactorial process--First American Cancer Society Award Lecture on Cancer Epidemiology and Prevention. Cancer Res 1992:52:6735-40.

36 Song M, Camargo MC, Weinstein SJ, et al. Family history of cancer in first-degree relatives and risk of gastric cancer and its precursors in a Western population. Gastric Cancer 2018;21:729-37.

37 Ferro A, Morais S, Rota M, et al. Tobacco smoking and gastric cancer: meta-analyses of published data versus pooled analyses of individual participant data (StoP Project). Eur I Cancer Prev 2018;27:197-204.

38 Han F, Wang X, Wang X, et al. Meta-analysis of the association of CYP1A1 polymorphisms with gastric cancer susceptibility and interaction with tobacco smoking. Mol Biol Rep 2012:39:8335-44.

39 La Torre G, Chiaradia G, Gianfagna F, et al. Smoking status and gastric cancer risk: an updated meta-analysis of case-control studies published in the past ten years. Tumori 2009:95:13-22.

40 Ladeiras-Lopes R, Pereira AK, Nogueira A, et al. Smoking and gastric cancer: systematic review and meta-analysis of cohort studies. Cancer Causes Control 2008;19:689-701.

41 Ma L, Chow JY, Cho CH. Effects of cigarette smoking on gastric ulcer formation and healing: possible mechanisms of action. J Clin Gastroentero/1998;27(Suppl 1):S80-6. 
42 Li LF, Chan RL, Lu L, et al. Cigarette smoking and gastrointestinal diseases: the causal relationship and underlying molecular mechanisms (review). Int J Mol Med 2014;34:372-80.

43 Bornschein J, Dingwerth A, Selgrad M, et al. Adenocarcinomas at different positions at the gastro-oesophageal junction show distinct association with gastritis and gastric preneoplastic conditions. Eur J Gastroenterol Hepatol 2015;27:492-500.

44 Siewert JR, Stein HJ. Classification of adenocarcinoma of the oesophagogastric junction. Br J Surg 1998:85:1457-9.

45 Fenton JJ, Abraham L, Taplin SH, et al. Effectiveness of computer-aided detection in community mammography practice. J Natl Cancer Inst 2011;103:1152-61.

46 Fenton JJ, Taplin SH, Carney PA, et al. Influence of computer-aided detection on performance of screening mammography. N Engl J Med 2007;356:1399-409.

47 van Rossum LG, van Rijn AF, Laheij RJ, et al. Random comparison of guaiac and immunochemical fecal occult blood tests for colorectal cancer in a screening population. Gastroenterology 2008;135:82-90.

48 Dancourt V, Lejeune C, Lepage C, et al. Immunochemical faecal occult blood tests are superior to guaiac-based tests for the detection of colorectal neoplasms. Eur J Cancer 2008;44:2254-8.
49 Brenner H, Tao S. Superior diagnostic performance of faecal immunochemical tests for haemoglobin in a head-to-head comparison with guaiac based faecal occult blood test among 2235 participants of screening colonoscopy. Eur J Cancer 2013;49:3049-54.

$50 \mathrm{Hol}$ L, Wilschut JA, van Ballegooijen M, et al. Screening for colorectal cancer: random comparison of guaiac and immunochemical faecal occult blood testing at different cut-off levels. Br J Cancer 2009;100:1103-10.

51 Grubb RL, Pinsky PF, Greenlee RT, et al. Prostate cancer screening in the Prostate, Lung, Colorectal and Ovarian cancer screening trial: update on findings from the initial four rounds of screening in a randomized trial. BJU Int 2008;102:1524-30.

52 Rugge M, Meggio A, Pennelli G, et al. Gastritis staging in clinical practice: the OLGA staging system. Gut 2007;56:631-6.

53 Yue H, Shan L, Bin L. The significance of OLGA and OLGIM staging systems in the risk assessment of gastric cancer: a systematic review and meta-analysis. Gastric Cancer 2018:21:579-87.

54 Wang X, Lu B, Meng L, et al. The correlation between histological gastritis staging'OLGA/OLGIM' and serum pepsinogen test in assessment of gastric atrophy/ intestinal metaplasia in China. Scand J Gastroenterol 2017;52:822-7. 